\title{
THE WEYL CALCULUS FOR HERMITIAN MATRICES
}

\author{
BRIAN JEFFERIES
}

(Communicated by Palle E. T. Jorgensen)

\begin{abstract}
The Weyl calculus is a means of constructing functions of a system of hermitian operators which do not necessarily commute with each other. This note gives a new proof of a formula, due to E. Nelson, for the Weyl calculus associated with a system of hermitian matrices.
\end{abstract}

\section{INTRODUCTION}

Given a collection of $d$ selfadjoint operators $A_{1}, \ldots, A_{d}$ acting on $\mathcal{H}=\mathbb{C}^{n}$, or equivalently, a collection of $n \times n$ hermitian matrices, the Weyl functional calculus [A], [T] for the $d$-tuple $A=\left(A_{1}, \ldots, A_{d}\right)$ is an $\mathcal{L}(\mathcal{H})$-valued distribution which represents a particular rule allowing the construction of certain functions of the matrices $A_{1}, \ldots, A_{d}$. The matrices $A_{1}, \ldots, A_{d}$ do not necessarily commute with each other, so there is no fundamentally unique way of forming such functions. However, the Weyl functional calculus is determined by a few natural conditions; see $[\mathrm{A}$, Theorem 2.4].

For $\xi=\left(\xi_{1}, \ldots, \xi_{d}\right) \in \mathbb{R}^{d}$, the matrix $\langle\xi, A\rangle=\sum_{j=1}^{d} \xi_{j} A_{j}$ is again hermitian and hence $\left\|e^{i\langle\xi, A\rangle}\right\|=1$ in the space $\mathcal{L}(\mathcal{H})$ of all operators on $\mathcal{H}$ endowed with the uniform operator norm. Let $\mathcal{S}\left(\mathbb{R}^{d}\right)$ denote the Schwartz space of $\mathbb{C}$-valued, rapidly decreasing functions on $\mathbb{R}^{d}$. More precisely, the Weyl calculus for $A$ is the $\mathcal{L}(\mathcal{H})$-valued distribution $\mathcal{W}_{A}$ defined by

$$
\mathcal{W}_{A}(f)=(2 \pi)^{-d / 2} \int_{\mathbb{R}^{d}} e^{i\langle\xi, A\rangle} \hat{f}(\xi) d \xi \quad \text { for all } f \in \mathcal{S}\left(\mathbb{R}^{d}\right) .
$$

The Fourier transform $\hat{f}$ of a function $f$ integrable over $\mathbb{R}^{d}$ is defined by

$$
\hat{f}(\xi)=(2 \pi)^{-d / 2} \int_{\mathbb{R}^{d}} e^{-i\langle x, \xi\rangle} f(x) d x
$$

for all $\xi \in \mathbb{R}^{d}$ and $\check{f}(x)=(2 \pi)^{-d / 2} \int_{\mathbb{R}^{d}} e^{i\langle x, \xi\rangle} f(x) d x, x \in \mathbb{R}^{d}$, denotes the inverse Fourier transform. In terms of distribution theory, $\mathcal{W}_{A}$ is the Fourier transform of the matrix-valued distribution

$$
f \mapsto(2 \pi)^{-d / 2} \int_{\mathbb{R}^{d}} e^{i\langle\xi, A\rangle} f(\xi) d \xi, \quad f \in \mathcal{S}\left(\mathbb{R}^{d}\right) .
$$

Received by the editors July 5, 1994.

1991 Mathematics Subject Classification. Primary 47A60, 47B15; Secondary 35E05, 15A60.

Key words and phrases. Functional calculus, Weyl calculus, hermitian matrix, distribution. 
Many of the results concerning the Weyl calculus in the Hilbert space setting (relevant to the present note) were established in [T], but for convenience, references are made to the later work of Anderson [A], who worked with Banach spaces. In particular, by the Payley-Wiener theorem, $\mathcal{W}_{A}$ is the restriction to $\mathcal{S}\left(\mathbb{R}^{d}\right)$ of a unique matrix-valued distribution defined on the space $C^{\infty}\left(\mathbb{R}^{d}\right)$ of all smooth functions on $\mathbb{R}^{d}[\mathrm{~A}$, Lemma 2.3]. There is no harm in using the same symbol to denote this extension. If $\mathbf{x}_{1}, \mathbf{x}_{2}$ denote the projections of $\mathbb{R}^{d}$ onto the first and second coordinates, respectively, it turns out that $\mathcal{W}_{A}\left(\mathbf{x}_{1} \mathbf{x}_{2}\right)=\frac{1}{2}\left(A_{1} A_{2}+A_{2} A_{1}\right)$. In general, polynomials on $\mathbb{R}^{d}$ are mapped by the Weyl functional calculus into the corresponding polynomials in the $d$-tuple $A$ of matrices, but with products suitably symmetrised [A, Theorem 2.4].

In the finite-dimensional setting, E. Nelson [N, Theorem 9] gave an explicit formula for the Weyl calculus. As pointed out in [A, p. 241], this amounts to calculating the fundamental solution for a hyperbolic system of partial differental equations. The purpose of this note is to provide another proof of Nelson's formula. The essential ingredients of the proof follow.

As is well known from matrix theory, a function of an $n \times n$ matrix $M$ can be expressed as a polynomial in $M$ of degree less than $n$. The key to calculating the Weyl calculus in the finite-dimensional setting is to find a suitable expression

$$
e^{i M}=\gamma_{0}(M)+\gamma_{1}(M) M+\cdots+\gamma_{n-1}(M) M^{n-1}
$$

for all $n \times n$ hermitian matrices $M$. This was achieved in the proof of [N, Theorem 9] by an ingenious application of recursion relations and induction. The proof below is more pedestrian. The Cayley-Hamilton theorem and binomial expansions are invoked to find the expression

$$
(\zeta I-M)^{-1}=\alpha_{0}(M, \zeta)+\alpha_{1}(M, \zeta) M+\cdots+\alpha_{n-1}(M, \zeta) M^{n-1}
$$

for all complex numbers $\zeta$ lying outside the spectrum $\sigma(M)$ of $M$. By applying the formula $e^{i M}=\frac{1}{2 \pi i} \int_{C} e^{i \zeta}(\zeta I-M)^{-1} d \zeta$, obtained from the Riesz calculus for a simple closed curve $C$ about $\sigma(M)$, the representation (1) follows.

As mentioned earlier, the Weyl calculus $\mathcal{W}_{A}$ is $(2 \pi)^{-d / 2}$ times the Fourier transform of the matrix-valued function $\xi \mapsto e^{i\langle A, \xi\rangle}, \xi \in \mathbb{R}^{d}$, in the sense of distributions. To calculate $\mathcal{W}_{A}$, it is then necessary to obtain the Fourier transform of the function (1) in the case that $M=\langle A, \xi\rangle$. The representation

$$
\frac{1}{2 \pi i} \int_{C} \frac{f(\zeta)}{\operatorname{det}(\zeta I-M)} d \zeta=\frac{1}{(n-1) !} \int_{\Sigma} f^{(n-1)}(\langle M u, u\rangle) d \nu(u)
$$

facilitates this calculation. Here $f$ is a function analytic in a neighbourhood of the convex hull $\operatorname{co}(\sigma(M))$ of $\sigma(M), C$ is a simple closed curve about $\sigma(M)$ and $\nu$ is the unitarily invariant probability measure on the unit sphere $\Sigma$ in $\mathbb{C}^{n}$. Only the case $f(\zeta)=e^{i \zeta}$ is needed in the calculation of $\mathcal{W}_{A}$, but the general formula is proved in the proposition below.

\section{Nelson's formula for the Weyl Calculus}

In order to state Nelson's formula for the Weyl calculus, we need to fix some terminology. Let $A=\left(A_{1}, \ldots, A_{d}\right)$ be a $d$-tuple of $n \times n$ hermitian matrices. Let 
$\nu$ be the unitarily invariant probability measure on the unit sphere $\Sigma=\left\{u \in \mathbb{C}^{n}\right.$ : $|u|=1\}$ in $\mathbb{C}^{n}$. Let $W_{A}: u \mapsto\left(\left\langle A_{1} u, u\right\rangle, \ldots,\left\langle A_{d} u, u\right\rangle\right) \in \mathbb{R}^{d}$, for all $u \in \Sigma$. Then

$$
\int_{\Sigma} e^{i\langle\langle A, \xi\rangle u, u\rangle} d \nu(u)=\int_{\mathbb{R}^{d}} e^{i\langle x, \xi\rangle} d \nu \circ W_{A}^{-1}(x) .
$$

Here $\langle x, \xi\rangle=\sum_{j=1}^{d} x_{j} \xi_{j}$ is the inner product of $\mathbb{R}^{d}$. Let $\mu_{A}=\nu \circ W_{A}^{-1}$, and set $\check{\mu}_{A}(\xi)=(2 \pi)^{-d / 2} \int_{\mathbb{R}^{d}} e^{i\langle x, \xi\rangle} d \mu_{A}(x)$ for all $\xi \in \mathbb{R}^{d}$.

The space of smooth functions on $\mathbb{R}^{d}$ is denoted by $C^{\infty}\left(\mathbb{R}^{d}\right)$ and the smooth functions on $\mathbb{R}^{d}$ with compact support by $C_{c}^{\infty}\left(\mathbb{R}^{d}\right)$. If $T$ is a matrix-valued distribution on $\mathcal{S}\left(\mathbb{R}^{d}\right)$, then the Fourier transform $\hat{T}$ of $T$ is defined by $\hat{T}(f)=T(\hat{f})$ for all $f \in \mathcal{S}\left(\mathbb{R}^{d}\right)$. Similarly, the inverse Fourier transform $\check{T}$ of $T$ is defined by $\check{T}(f)=T(\check{f})$ for all $f \in \mathcal{S}\left(\mathbb{R}^{d}\right)$. For a distribution on $\mathbb{C}^{\infty}\left(\mathbb{R}^{d}\right), \hat{T}$ is used to denote the Fourier transform of the restriction of $T$ to $\mathcal{S}\left(\mathbb{R}^{d}\right)$. As is customary, a function $\phi$ is confounded with the distribution $T_{\phi}: f \mapsto \int_{\mathbb{R}^{d}} f(x) \phi(x) d x$ it defines. Thus, $\check{\mu}_{A}$ is the inverse Fourier transform of $\mu_{A}$ in the sense of distributions.

For any $n \times n$ matrix $M$ and $k=1, \ldots, n$, let $\phi_{k}(M)$ be the sum of the principal minors in $M$ of order $k$ and set $\phi_{0}(M)=1$. The same expression is adopted if $M$ is a matrix differential operator whose entries are complex linear combinations of the partial differential operators $\partial / \partial x_{1}, \ldots, \partial / \partial x_{d}$ acting on the space of distributions $C^{\infty}\left(\mathbb{R}^{d}\right)^{\prime}$. Of particular interest is the matrix differential operator $M=\langle A, \nabla\rangle$ defined by $\langle A, \nabla\rangle=\sum_{j=1}^{d} A_{j} \partial / \partial x_{j}$. Because $\left(\xi_{j} f(\xi)\right)^{\prime}(x)=i \partial / \partial x_{j} \hat{f}(x)$ for all $f \in \mathcal{S}\left(\mathbb{R}^{d}\right)$, it follows that

$$
\left(\phi_{k}(\langle A, \xi\rangle) \check{T}(\xi)\right)^{\wedge}=\phi_{k}(i\langle A, \nabla\rangle) T
$$

for every distribution $T \in C^{\infty}\left(\mathbb{R}^{d}\right)^{\prime}$ whose inverse Fourier transform $\check{T}$ is a locally integrable function $\xi \mapsto \check{T}(\xi)$ with at most polynomial growth at infinity. Moreover

$$
\left(\xi \cdot \nabla_{\xi} \check{T}(\xi)\right)^{\wedge}=-\nabla \cdot \operatorname{id} T,
$$

where $\nabla \cdot$ id is the operator on $C_{c}^{\infty}\left(\mathbb{R}^{d}\right)^{\prime}$ defined on the dense subspace $C_{c}^{\infty}\left(\mathbb{R}^{d}\right)$ by $(\nabla \cdot \operatorname{id} f)(x)=\left(\nabla_{x} \cdot x\right) f(x)$ for all $f \in C_{c}^{\infty}\left(\mathbb{R}^{d}\right)$.

The remainder of the present note is devoted to a proof of the following result of E. Nelson [N, Theorem 9].

Theorem. Let $A=\left(A_{1}, \ldots, A_{d}\right)$ be a d-tuple of $n \times n$ hermitian matrices. The Weyl calculus for the d-tuple $A$ is given by

$$
\begin{aligned}
\mathcal{W}_{A}=\sum_{k=0}^{n-1} \sum_{j=0}^{n-k-1} \sum_{m=0}^{j}( & -1)^{k+m}\left(\begin{array}{c}
j \\
m
\end{array}\right) \frac{1}{(n-1-j+m) !} \\
& \times\langle A, \nabla\rangle^{k} \phi_{n-j-k-1}(\langle A, \nabla\rangle)(\nabla \cdot \mathrm{id})^{m} \mu_{A} .
\end{aligned}
$$

Remarks. (i) The statement of [N, Theorem 9] is essentially concerned with the situation in which $A$ is a basis of the real vector space of all $n \times n$ hermitian matrices, so that $d=n^{2}$. However, general properties of the Weyl calculus enable a derivation of the statement above from [N, Theorem 9] for other values of $d$.

(ii) The operator $\nabla$ should be replaced by $-\nabla$ in equation (16) of $[\mathrm{N}]$, because $-\nabla$ is the operator corresponding to multiplication of the inverse Fourier transform by $i \lambda$ in $(24)$ of $[\mathrm{N}]$. The following example verifies this observation. 
Example. Let $d=3$ and consider the Pauli matrices

$$
\sigma_{1}=\left(\begin{array}{cc}
0 & 1 \\
1 & 0
\end{array}\right), \quad \sigma_{2}=\left(\begin{array}{cc}
0 & -i \\
i & 0
\end{array}\right), \quad \sigma_{3}=\left(\begin{array}{cc}
1 & 0 \\
0 & -1
\end{array}\right) .
$$

Set $\sigma=\left(\sigma_{1}, \sigma_{2}, \sigma_{3}\right)$. By the theorem above,

$$
\mathcal{W}_{\sigma}=\mu_{\sigma}-(\nabla \cdot \mathrm{id}) \mu_{\sigma}-\langle\sigma, \nabla\rangle \mu_{\sigma}+\phi_{1}(\langle\sigma, \nabla\rangle) \mu_{\sigma} .
$$

Because $\phi_{1}(\langle\sigma, \nabla\rangle)=\operatorname{trace}(\langle\sigma, \nabla\rangle)=0$, the matrix $\mathcal{W}_{\sigma}(f)$ is given for every $f \in$ $C^{\infty}\left(\mathbb{R}^{3}\right)$ by

$$
\mathcal{W}_{\sigma}(f)=I \int_{S_{1}}(f+n \cdot \nabla f) d \mu_{1}+\int_{S_{1}}\langle\sigma, \nabla\rangle f d \mu_{1} .
$$

Here $S_{1}=\left\{x \in \mathbb{R}^{3}:|x|=1\right\}$ is the sphere of radius one centered at zero in $\mathbb{R}^{3}$, $\mu_{1}=\mu_{\sigma}$ is the unit surface measure on $S_{1}$ and $n(x)$ is the outward unit normal at $x \in S_{1}$. Thus, $\operatorname{supp} \mathcal{W}_{\sigma}=S_{1}$. This formula appears in [A, Theorem 4.1] by a direct calculation.

Let $M$ be an $n \times n$ matrix. The characteristic polynomial $p_{M}$ of $M$ is defined by $p_{M}(z)=\operatorname{det}(M-z I)$ for all $z \in \mathbb{C}$. For each $n \times n$ matrix $M$, let the complex numbers $a_{0}(M), \ldots, a_{n}(M)$ be the coefficients of the characteristic polynomial $p_{M}$ of $M$.

Given $\zeta \in \mathbb{C}$, the characteristic polynomial $p_{M-\zeta I}$ of $M-\zeta I$ is given by $p_{M-\zeta I}(z)$ $=\operatorname{det}(M-\zeta I-z I)=p_{M}(z+\zeta)$ for all $z \in \mathbb{C}$, so that

$$
p_{M-\zeta I}(z)=\sum_{h=0}^{n} a_{h}(M)(z+\zeta)^{h}=\sum_{h=0}^{n} a_{h}(M) \sum_{l=0}^{h}\left(\begin{array}{c}
h \\
l
\end{array}\right) \zeta^{h-l} z^{l} .
$$

By the Cayley-Hamilton theorem, $p_{M-\zeta I}(M-\zeta I)=0$, so if $\zeta$ is not an eigenvalue, then upon multiplying the resulting equation by $(M-\zeta I)^{-1}$ and rearranging the sums, we obtain

$$
\begin{aligned}
(M- & \zeta I)^{-1} \\
& =-p_{M}(\zeta)^{-1} \sum_{h=1}^{n} a_{h}(M) \sum_{l=1}^{h}\left(\begin{array}{c}
h \\
l
\end{array}\right) \zeta^{h-l}(M-\zeta I)^{l-1} \\
& =p_{M}(\zeta)^{-1} \sum_{k=0}^{n-1}\left(\sum_{l=k+1}^{n}(-1)^{l-k} \sum_{h=l}^{n} a_{h}(M)\left(\begin{array}{c}
h \\
l
\end{array}\right)\left(\begin{array}{c}
l-1 \\
k
\end{array}\right) \zeta^{h-k-1}\right) M^{k} .
\end{aligned}
$$

For each $k=0, \ldots, n-1$, let $g=l-k-1$ in the sum

$$
S_{k}=\sum_{l=k+1}^{n}(-1)^{l-k} \sum_{h=l}^{n} a_{h}(M)\left(\begin{array}{c}
h \\
l
\end{array}\right)\left(\begin{array}{c}
l-1 \\
k
\end{array}\right) \zeta^{h-k-1},
$$

and interchange the sums over $g$ and $h$ to get

$$
S_{k}=-\sum_{h=k+1}^{n} \sum_{g=0}^{h-k-1}(-1)^{g} a_{h}(M)\left(\begin{array}{c}
h \\
g+k+1
\end{array}\right)\left(\begin{array}{c}
g+k \\
k
\end{array}\right) \zeta^{h-k-1} .
$$

With the substitution $j=h-k-1$, we have

$$
S_{k}=-\sum_{j=0}^{n-k-1} a_{j+k+1}(M) \zeta^{j}\left(\sum_{g=0}^{j}(-1)^{g}\left(\begin{array}{c}
j+k+1 \\
g+k+1
\end{array}\right)\left(\begin{array}{c}
g+k \\
k
\end{array}\right)\right) .
$$


Lemma. For all $j, k=0,1, \ldots$,

$$
\sum_{g=0}^{j}(-1)^{g}\left(\begin{array}{c}
j+k+1 \\
g+k+1
\end{array}\right)\left(\begin{array}{c}
g+k \\
k
\end{array}\right)=1 .
$$

Proof. Let $j, k=0,1, \ldots$, and set $f(x)=\left((1-x)^{j+k+1}-1\right) / x$. Upon expanding the power and differentiating $k$ times, we get

$$
\sum_{g=0}^{j}(-1)^{g}\left(\begin{array}{c}
j+k+1 \\
g+k+1
\end{array}\right)\left(\begin{array}{c}
g+k \\
k
\end{array}\right)=\frac{(-1)^{k+1}}{k !} f^{(k)}(1) .
$$

By Leibniz's formula for the differentiation of products,

$$
f^{(k)}(x)=\sum_{m=0}^{k}\left(\begin{array}{c}
k \\
m
\end{array}\right)(-1)^{k-m}(k-m) ! x^{-(k-m+1)} \frac{d^{m}}{d x^{m}}\left((1-x)^{j+k+1}-1\right) .
$$

The only non-zero term in $f^{(k)}(1)$ is the one with $m=0$, so $f^{(k)}(1)=(-1)^{k+1} k$ ! .

It follows that $(\zeta I-M)^{-1}=\sum_{k=0}^{n-1}\left(\sum_{j=0}^{n-k-1} a_{j+k+1}(M) \zeta^{j} / p_{M}(\zeta)\right) M^{k}$. Alternatively, the equality $p_{M}(\zeta)=(\zeta I-M) \sum_{k=0}^{n-1}\left(\sum_{j=0}^{n-k-1} a_{j+k+1}(M) \zeta^{j}\right) M^{k}$ is easily verified from the Cayley-Hamilton theorem.

Now suppose that $C$ is a simple closed curved in $\mathbb{C}$ surrounding the set $\sigma(M)$ of eigenvalues of the $n \times n$ matrix $M$. Then by the Riesz functional calculus,

$$
\begin{aligned}
e^{i M} & =\frac{1}{2 \pi i} \int_{C} e^{i \zeta}(\zeta I-M)^{-1} d \zeta \\
& =\frac{1}{2 \pi i} \sum_{k=0}^{n-1}\left(\sum_{j=0}^{n-k-1} a_{j+k+1}(M) \int_{C} \frac{e^{i \zeta} \zeta^{j}}{p_{M}(\zeta)} d \zeta\right) M^{k} .
\end{aligned}
$$

Now for all $t \in \mathbb{R}$ so close to one that $C$ surrounds $t \sigma(M)$ too,

$$
\begin{aligned}
\int_{C} \frac{e^{i t \zeta}(i \zeta)^{j}}{p_{M}(\zeta)} d \zeta & =\frac{\partial^{j}}{\partial t^{j}} \int_{C} \frac{e^{i t \zeta}}{p_{M}(\zeta)} d \zeta \\
& =\frac{\partial^{j}}{\partial t^{j}} t^{n-1} \int_{C} \frac{e^{i \zeta}}{p_{t M}(\zeta)} d \zeta .
\end{aligned}
$$

An appeal to Leibniz's formula for the differentiation of products yields

$$
\begin{aligned}
e^{i M}=\frac{1}{2 \pi i} \sum_{k=0}^{n-1} M^{k} & \sum_{j=0}^{n-k-1} i^{-j} a_{j+k+1}(M) \\
& \times \sum_{m=0}^{j}\left(\begin{array}{c}
j \\
m
\end{array}\right) \frac{(n-1) !}{(n-1-j+m) !}\left[\frac{\partial^{m}}{\partial t^{m}} \int_{C} \frac{e^{i z}}{p_{t M}(z)} d z\right]_{t=1} .
\end{aligned}
$$

To calculate the Fourier transform of (2) with $M=\langle A, \xi\rangle$, the following observation is useful. 
Proposition. Let $\nu$ be the unitarily invariant probability measure on the unit sphere $\Sigma$ in $\mathbb{C}^{n}$. Let $M$ be a normal $n \times n$ matrix, and let $U$ be a simply connected open subset of $\mathbb{C}$ containing the convex hull $\operatorname{co}(\sigma(M))$ of the spectrum $\sigma(M)$ of $M$.

Let $C$ be a simple closed curve around $\sigma(M)$ contained in $U$, and suppose that $f: U \rightarrow \mathbb{C}$ is analytic. Then

$$
\frac{1}{2 \pi i} \int_{C} \frac{f(\zeta)}{p_{M}(\zeta)} d \zeta=\frac{(-1)^{n}}{(n-1) !} \int_{\Sigma} f^{(n-1)}(\langle M u, u\rangle) d \nu(u)
$$

Proof. Suppose first that $x \in \mathbb{C}, x \neq 0$ and $f(z)=e^{-x z}$ for all $z \in \mathbb{C}$. If $\lambda_{1}, \ldots, \lambda_{n}$ are distinct complex numbers and $M$ is the diagonal matrix with entries $\lambda_{1}, \ldots, \lambda_{n}$, then $f(\langle M u, u\rangle)=\exp \left(-x \sum_{j=1}^{n} \lambda_{j}\left|u_{j}\right|^{2}\right)$ for $u=\left(u_{1}, \ldots, u_{n}\right) \in \mathbb{C}^{n}$ and

$$
\begin{aligned}
\frac{1}{2 \pi i} \int_{C} \frac{f(\zeta)}{p_{M}(\zeta)} d \zeta & =\frac{1}{2 \pi i} \int_{C} \frac{f(\zeta)}{\left(\lambda_{1}-\zeta\right) \cdots\left(\lambda_{n}-\zeta\right)} d \zeta \\
& =\frac{(-1)^{n}}{2 \pi i} \sum_{k=1}^{n} \frac{1}{\prod_{j \neq k}\left(\lambda_{k}-\lambda_{j}\right)} \int_{C} \frac{f(\zeta)}{\zeta-\lambda_{k}} d \zeta \\
& =(-1)^{n} \sum_{k=1}^{n} \frac{e^{-x \lambda_{k}}}{\prod_{j \neq k}\left(\lambda_{k}-\lambda_{j}\right)}
\end{aligned}
$$

Let $\Delta_{n}=\left\{w \in \mathbb{R}^{n}: w_{j} \geq 0, \sum_{j=1}^{n} w_{j}=1\right\}$ be the unit simplex in $\mathbb{R}^{n}$, and denote the normalised Lebesgue measure on $\Delta_{n}$ by $\sigma$. Then, as argued in [N, p. 186], $\sigma$ is the image of the measure $\nu$ under the map $u \mapsto\left(\left|u_{1}\right|^{2}, \ldots,\left|u_{n}\right|^{2}\right), u \in \mathbb{C}^{n}$. The measure $\frac{1}{(n-1) !} \sigma$ is the image of the Lebesgue measure on the set $\left\{0 \leq t_{1} \leq\right.$ $\left.\cdots \leq t_{n-1} \leq 1\right\}$ by the affine bijection $w_{j}=t_{j}-t_{j-1}, j=1, \ldots, n$, with $t_{0}=0$ and $t_{n}=1$, so by the change of variables formula,

$$
\begin{aligned}
\frac{1}{(n-1) !} & \int_{\Sigma} \exp \left(-x \sum_{j=1}^{n} \lambda_{j}\left|u_{j}\right|^{2}\right) d \nu(u)=\frac{1}{(n-1) !} \int_{\Delta_{n}} \exp \left(-x \sum_{j=1}^{n} \lambda_{j} w_{j}\right) d \sigma(w) \\
& =e^{-x \lambda_{n}} \int_{0}^{1} \int_{0}^{t_{n-1}} \cdots \int_{0}^{t_{2}} e^{x \sum_{j=1}^{n-1}\left(\lambda_{j+1}-\lambda_{j}\right) t_{j}} d t_{1} \cdots d t_{n-1} \\
& =\frac{(-1)^{n-1}}{x^{n-1}} \sum_{k=1}^{n} \frac{e^{-x \lambda_{k}}}{\prod_{j \neq k}\left(\lambda_{k}-\lambda_{j}\right)} .
\end{aligned}
$$

Combined with the preceding calculation, we obtain

$$
\frac{1}{2 \pi i} \int_{C} \frac{f(\zeta)}{p_{M}(\zeta)} d \zeta=-\frac{x^{n-1}}{(n-1) !} \int_{\Sigma} \exp \left(-x \sum_{j=1}^{n} \lambda_{j}\left|u_{j}\right|^{2}\right) d \nu(u) .
$$

The continuity of both sides in $\lambda=\left(\lambda_{1}, \ldots, \lambda_{n}\right)$ ensures the equality (4) for all $\lambda \in \mathbb{C}^{n}$ with $M=\operatorname{diag}\left(\lambda_{1}, \ldots, \lambda_{n}\right)$.

Now suppose that $q$ is a polynomial, $\phi: \mathbb{R} \rightarrow \mathbb{R}$ is a function of the form $\phi(x)=q(x) e^{-x^{2} / 2}, x \in \mathbb{R}$, and $f(z)=\int_{\mathbb{R}} e^{-i x z} \phi(x) d x$ for all $z \in \mathbb{C}$. Then the 
equality (3) is valid for $f$ by Fubini's theorem and equation (4), which is also valid with $x \in \mathbb{R}$ replaced by $i x$.

The set $U$ is simply connected, so by Runge's theorem [R, Theorem13.11] an analytic function on $U$ can be approximated uniformly on any compact subset of $U$ by polynomials, and so also by functions of the form $z \mapsto p(z) e^{-z^{2} / 2}, z \in \mathbb{C}$, with $p$ a polynomial. Any such function is (the analytic continuation of) the Fourier transform of a function of the form $\phi$ above.

Thus, the set of all functions $f$ for which (3) is true is dense in the space $H(U)$ of functions analytic in the set $U$, with respect to the topology of uniform convergence on compact subsets of $U$. Therefore, (3) is true for all $f \in H(U)$ and all diagonal matrices $M$. Both sides of (3) are unchanged if $M$ is replaced by $U M U^{*}$ for a unitary transformation $U$ of $\mathbb{C}^{n}$, so (3) is valid for all normal matrices $M$.

If we apply the proposition to the function $f(z)=e^{i z}, z \in \mathbb{C}$, and the hermitian matrix $M=\langle A, \xi\rangle$ and note, as mentioned earlier, that

$$
\check{\mu}_{A}(\xi)=(2 \pi)^{-d / 2} \int_{\Sigma} e^{i\langle\langle A, \xi\rangle u, u\rangle} d \nu(u)
$$

then it follows that for all $\xi \in \mathbb{R}^{d}$

$$
\frac{1}{2 \pi i} \int_{C} \frac{e^{i z}}{p_{\langle A, \xi\rangle}(z)} d z=-\frac{(-i)^{n-1}(2 \pi)^{d / 2}}{(n-1) !} \check{\mu}_{A}(\xi) .
$$

The equality $\left[\frac{d}{d t} g(t x)\right]_{t=1}=x \cdot \nabla_{x} g(x)$ is valid for all differentiable functions $g$ on $\mathbb{R}^{d}$, so for $M=\langle A, \xi\rangle$, equation (2) becomes

$$
\begin{aligned}
e^{i\langle A, \xi\rangle}=(2 \pi)^{d / 2}(-1)^{n} \sum_{k=0}^{n-1}\langle A, \xi\rangle^{k} \sum_{j=0}^{n-k-1} i^{n-j-1} a_{j+k+1}(\langle A, \xi\rangle) \\
\times \sum_{m=0}^{j}\left(\begin{array}{c}
j \\
m
\end{array}\right) \frac{\left(\xi \cdot \nabla_{\xi}\right)^{m} \check{\mu}_{A}(\xi)}{(n-1-j+m) !} .
\end{aligned}
$$

The coefficients of the characteristic polynomial of an $n \times n$ matrix $M$ are calculated from the sums of the principal minors by virtue of the equality $a_{s}(M)=$ $(-1)^{s} \phi_{n-s}(M)$, for all $s=0, \ldots, n-1$. If we substitute for $a_{s}(M), s=1, \ldots, n-1$, in (5) and note that $\mathcal{W}_{A}$ is $(2 \pi)^{-d / 2}$ times the Fourier transform of the distribution defined by the right-hand side of (5), then it follows from the discussion at the beginning of this section that the Weyl calculus for the $d$-tuple $A$ is given by

$$
\begin{aligned}
\mathcal{W}_{A}=(-1)^{n} \sum_{k=0}^{n-1} \sum_{j=0}^{n-k-1} \sum_{m=0}^{j}(-1)^{j+k+1} i^{n-j-1}\left(\begin{array}{c}
j \\
m
\end{array}\right) \frac{1}{(n-1-j+m) !} \\
\quad \times(i\langle A, \nabla\rangle)^{k} \phi_{n-j-k-1}(i\langle A, \nabla\rangle)(-\nabla \cdot \mathrm{id})^{m} \mu_{A} \\
=\sum_{k=0}^{n-1} \sum_{j=0}^{n-k-1} \sum_{m=0}^{j}(-1)^{k+m}\left(\begin{array}{c}
j \\
m
\end{array}\right) \frac{1}{(n-1-j+m) !} \\
\times\langle A, \nabla\rangle^{k} \phi_{n-j-k-1}(\langle A, \nabla\rangle)(\nabla \cdot \mathrm{id})^{m} \mu_{A} .
\end{aligned}
$$




\section{REFERENCES}

[A] R.F.V. Anderson, The Weyl functional calculus, J. Funct. Anal. 4 (1969), 240-267. MR 58:30405

[N] E. Nelson, Operants: A functional calculus for non-commuting operators, Functional Analysis and Related Fields, Proceedings of a conference in honour of Professor Marshal Stone (Univ. of Chicago, May 1968) (F.E. Browder, ed.), Springer-Verlag, Berlin, Heidelberg, and New York, 1970, pp. 172-187. MR 54:978

[R] W. Rudin, Real and complex analysis, 2nd ed., McGraw-Hill, New York, 1987. MR 88k:00002

[T] M.E. Taylor, Functions of several self-adjoint operators, Proc. Amer. Math. Soc. 19 (1968), 91-98. MR 36:3149

School of Mathematics, University of New South Wales, New South Wales 2052, Australia

E-mail address: B.Jefferies@unsw.edu.au 